\title{
WEIGHTED NORM INEQUALITIES FOR VILENKIN-FOURIER SERIES
}

\author{
WO-SANG YOUNG
}

\begin{abstract}
Let $S_{n} f$ be the $n$th partial sum of the Vilenkin-Fourier series of $f \in L^{1}$. For $1<p<\infty$, we characterize all weight functions $w$ such that if $f \in L^{p}(w), S_{n} f$ converges to $f$ in $L^{p}(w)$. We also determine all weight functions $w$ such that $\left\{S_{n}\right\}$ is uniformly of weak type $(1,1)$ with respect to $w$.
\end{abstract}

\section{INTRODUCTION}

The Vilenkin-Fourier series is a generalization of the Walsh-Fourier series. While the Walsh functions are characters of the countable direct product of groups of order 2, the Vilenkin system consists of characters of $G=\prod_{i=0}^{\infty} Z_{p_{i}}$, a direct product of cyclic groups of order $p_{i}$, where $p_{i}$ is any integer $\geq 2$. In this paper, there is no restriction on the orders $\left\{p_{i}\right\}$.

Let $\mu$ be the Haar measure on $G$ normalized by $\mu(G)=1$. We identify $G$ with the unit interval $(0,1)$ in the following manner. Let $m_{0}=1, m_{k}=$ $\prod_{i=0}^{k-1} p_{i}, k=1,2, \ldots$ We associate with each $\left\{x_{i}\right\} \in G, 0 \leq x_{i}<p_{i}$, the point $\sum_{i=0}^{\infty} x_{i} m_{i+1}^{-1} \in(0,1)$. If we disregard the countable set of $p_{i}$-rationals, this mapping is one-one, onto and measure-preserving.

The characters of $G$ are the finite products of an orthonormal system $\left\{\phi_{k}\right\}$ of functions on $G$, defined by $\phi_{k}(x)=\exp \left(2 \pi i x_{k} / p_{k}\right), x=\left\{x_{k}\right\} \in G, k=$ $0,1, \ldots$. To enumerate the finite products of $\left\{\phi_{k}\right\}$, we write each nonnegative integer $n$ as a finite sum, $n=\sum_{k=0}^{\infty} \alpha_{k} m_{k}$, with $0 \leq \alpha_{k}<p_{k}$, and define $\chi_{n}=\prod_{k=0}^{\infty} \phi_{k}^{\alpha_{k}}$. The functions $\left\{\chi_{n}\right\}$ are the characters of $G$, and they form a complete orthonormal system on $G$.

For $f \in L^{1}$, let

$$
S_{n} f(x)=\int_{G} f(t) \sum_{j=0}^{n-1} \chi_{j}(x-t) d \mu(t), \quad n=1,2, \ldots,
$$

be the $n$th partial sum of the Vilenkin-Fourier series of $f$. In [10], we showed that for $f \in L^{p}, 1<p<\infty$,

$$
\lim _{n \rightarrow \infty} \int_{G}\left|S_{n} f-f\right|^{p} d \mu=0 .
$$

Received by the editors September 6, 1989 and, in revised form, September 5, 1991.

1991 Mathematics Subject Classification. Primary 42C10; Secondary 42A20, 42A50, 43A50. 
In this paper, we identify, for $1<p<\infty$, all weight functions $w$ such that for any $f \in L^{p}(w)$, we have $f \in L^{1}$ and

$$
\lim _{n \rightarrow \infty} \int_{G}\left|S_{n} f-f\right|^{p} w d \mu=0 .
$$

(Here $L^{p}(w)$ denotes the space of measurable functions on $G$ such that $\|f\|_{p, w}$ $\left.\equiv\left(\int_{G}|f|^{p} w d \mu\right)^{1 / p}<\infty.\right)$

In order to characterize these weight functions, we use the following notation. Let $\left\{G_{k}\right\}$ be the sequence of subgroups of $G$ defined by

$$
G_{0}=G, \quad G_{k}=\prod_{i=0}^{k-1}\{0\} \times \prod_{i=k}^{\infty} Z_{p_{i}}, \quad k=1,2, \ldots
$$

On the interval $(0,1)$, cosets of $G_{k}$ are intervals of the form $\left(\mathrm{jm}_{k}^{-1}\right.$, $\left.(j+1) m_{k}^{-1}\right), j=0,1, \ldots, m_{k}-1$. For $k=0,1, \ldots, j=1, \ldots, p_{k}$, let $I_{j k}$ be the set in $G_{k}$ that corresponds to the interval $\left(0, j m_{k+1}^{-1}\right)$. The collection of all translates of $I_{j k}$ in $G$, for all $j=1, \ldots, p_{k}, k=0,1, \ldots$, is denoted by $\mathscr{I}$. In other words, a set $I$ belongs to $\mathscr{I}$ if

(i) for some $x \in G, k=0,1, \ldots, I \subset x+G_{k}$,

(ii) $I$ is a union of cosets of $G_{k+1}$, and

(iii) if we consider $x+G_{k}$ as a circle, $I$ is an interval.

We shall call the sets in $\mathscr{I}$ generalized intervals.

We say that $w$ is a weight function on $G$ if $w$ is measurable and $0 \leq$ $w(x) \leq \infty$. We shall exclude the trivial cases $w \equiv 0$ a.e. and $w \equiv \infty$ a.e.

Our main results are as follows:

Theorem 1.1. Let $w$ be a weight function on $G$ and $1<p<\infty$. The following statements are equivalent:

(i) $w$ satisfies the $A_{p}(G)$ condition: there is a constant $C$ such that for every $I \in \mathscr{I}$,

$$
\left(\frac{1}{\mu(I)} \int_{I} w d \mu\right)\left(\frac{1}{\mu(I)} \int_{I} w^{-1 /(p-1)} d \mu\right)^{p-1} \leq C .
$$

(ii) There is a constant $C$, depending only on $w$ and $p$, such that for every $f \in L^{p}(w)$, we have $f \in L^{1}$ and

$$
\int_{G}\left|S_{n} f\right|^{p} w d \mu \leq C \int_{G}|f|^{p} w d \mu, \quad n=1,2, \ldots
$$

(iii) For every $f \in L^{p}(w)$, we have $f \in L^{1}$ and

$$
\lim _{n \rightarrow \infty} \int_{G}\left|S_{n} f-f\right|^{p} w d \mu=0 .
$$

For the case where the orders of cyclic groups are bounded, i.e., $\sup _{i} p_{i}<\infty$, J. Gosselin [5] defined the $A_{p}$ condition, $1<p<\infty$, as the one where (1.1) holds for all $I$ that are cosets of $G_{k}, k=0,1, \ldots$. For this case, our $A_{p}(G)$ condition and his $A_{p}$ condition are equivalent. This can be seen as follows. Suppose $w$ satisfies the $A_{p}$ condition in [5], and $I \in \mathscr{I}$. Then, for some $x \in G, k=0,1, \ldots, I \subset x+G_{k}$, and $I$ is a union of cosets of $G_{k+1}$. It 
follows that $\mu(I) \geq \mu\left(G_{k+1}\right)=p_{k}^{-1} \mu\left(G_{k}\right)$. Hence

$$
\begin{aligned}
& \left(\frac{1}{\mu(I)} \int_{I} w d \mu\right)\left(\frac{1}{\mu(I)} \int_{I} w^{-1 /(p-1)} d \mu\right)^{p-1} \\
& \quad \leq p_{k}^{p}\left(\frac{1}{\mu\left(G_{k}\right)} \int_{x+G_{k}} w d \mu\right)\left(\frac{1}{\mu\left(G_{k}\right)} \int_{x+G_{k}} w^{-1 /(p-1)} d \mu\right)^{p-1} \\
& \quad \leq C, \quad \text { if } \sup _{i} p_{i}<\infty .
\end{aligned}
$$

For the bounded order case, the implication (i) $\Rightarrow$ (ii) in Theorem 1.1 is a consequence of the result in [5]. For another proof, see [8].

We also have the following theorem for functions in $L^{1}(w)$.

Theorem 1.2. Let $w$ be a weight function on $G$. The following statements are equivalent:

(i) $w$ satisfies the $A_{1}(G)$ condition: there is a constant $C$ such that for every $I \in \mathcal{I}$,

$$
\frac{1}{\mu(I)} \int_{I} w d \mu \leq C \underset{I}{\operatorname{essinf}} w,
$$

where ess $\inf _{I} w=\inf \{t>0: \mu\{x \in I: w(x)<t\}>0\}$.

(ii) There is a constant $C$, depending only on $w$, such that for every $f \in$ $L^{1}(w)$, we have $f \in L^{1}$ and

$$
\int_{\left\{\left|S_{n} f\right|>y\right\}} w d \mu \leq C y^{-1} \int_{G}|f| w d \mu, \quad n=1,2, \ldots, y>0 .
$$

Our proofs of Theorems 1.1 and 1.2 consist of adapting the methods of Hunt, Muckenhoupt, and Wheeden [6] and Cordoba and Fefferman [2] to VilenkinFourier series. We recall some properties of Vilenkin-Fourier series. Let $S_{n}^{*} f=$ $\bar{\chi}_{n} S_{n}\left(f \chi_{n}\right)$ be the $n$th modified partial sum, $n=1,2, \ldots$. It is shown in [8] that if $n=\sum_{k=0}^{\infty} \alpha_{k} m_{k}, 0 \leq \alpha_{k}<p_{k}$, then

$$
S_{n}^{*} f=\sum_{k=0}^{\infty} S_{\alpha_{k} m_{k}}^{*} f
$$

and

$$
S_{\alpha_{k} m_{k}}^{*} f(x)=\frac{1}{\mu\left(G_{k}\right)} \int_{x+G_{k}} f(t) \phi_{k}^{-\alpha_{k}}(x-t)\left(\sum_{j=0}^{\alpha_{k}-1} \phi_{k}^{j}(x-t)\right) d \mu(t) .
$$

(If $\alpha_{k}=0$, we interpret the sum on the right as zero.) $S_{\alpha_{k} m_{k}}^{*} f$ can be expressed in terms of certain conjugate functions of $f$ and $f \phi_{k}^{\alpha_{k}}$, which are defined as follows. For $f \in L^{1}$ and $x=\left\{x_{k}\right\} \in G$, let the $k$ th conjugate function of $f$ be

$$
H_{k} f(x)=\frac{1}{2} \frac{1}{\mu\left(G_{k}\right)} \int_{\left(x+G_{k}\right) \cap\left\{x_{k} \neq t_{k}\right\}} f(t) \cot \left(\pi\left(x_{k}-t_{k}\right) / p_{k}\right) d \mu(t),
$$


$k=0,1, \ldots$. Then

$$
\begin{aligned}
S_{\alpha_{k} m_{k}}^{*} f(x)= & \frac{\alpha_{k}}{\mu\left(G_{k}\right)} \int_{\left(x+G_{k}\right) \cap\left\{x_{k}=t_{k}\right\}} f(t) d \mu(t) \\
& +\frac{1}{2} \phi_{k}^{-\alpha_{k}}(x) \frac{1}{\mu\left(G_{k}\right)} \int_{\left(x+G_{k}\right) \cap\left\{x_{k} \neq t_{k}\right\}} f(t) \phi_{k}^{\alpha_{k}}(t) d \mu(t) \\
& -\frac{1}{2} \frac{1}{\mu\left(G_{k}\right)} \int_{\left(x+G_{k}\right) \cap\left\{x_{k} \neq t_{k}\right\}} f(t) d \mu(t) \\
& +i \phi_{k}^{-\alpha_{k}}(x) H_{k}\left(f \phi_{k}^{\alpha_{k}}\right)(x)-i H_{k} f(x) .
\end{aligned}
$$

To estimate $S_{n}^{*} f$, we have to deal simultaneously with the conjugate functions of $f$ and $f \phi_{k}^{\alpha_{k}}, k=0,1, \ldots$ In order to do this, we make the following changes in the proofs of [6 and 2]. In the proof of the weak type $(1,1)$ result, we modify the function $b(t)$ in the Calderon-Zygmund decomposition to satisfy both $\int_{I} b d \mu=0$ and $\int_{I} b \phi_{k}^{\alpha_{k}} d \mu=0$, where $\alpha_{k}$ depends on $I$ and $n$. This modification of the Calderon-Zygmund decomposition was introduced in [10]. In the proof of the strong type $(p, p)$ result, the sharp function is modified as follows: the mean value of $f$ over $I$ is replaced by a function $f_{I, n}=A+B \phi_{k}^{-\alpha_{k}}\left(\alpha_{k}\right.$ depending on $I$ and $\left.n\right)$ that satisfies $\int_{I}\left(f-f_{I, n}\right) d \mu=0$ and $\int_{I}\left(f-f_{I, n}\right) \phi_{k}^{\alpha_{k}} d \mu=0$. These modified sharp functions will be discussed in $\S 3$.

Section 2 contains some properties of $A_{p}(G)$. We also show that the $A_{p}(G)$ condition is necessary and sufficient for the $L^{p}(w)$-boundedness of a HardyLittlewood type maximal function that is appropriate for the study of VilenkinFourier series.

In $\S 3$ we introduce the modified sharp functions. We obtain estimates for the modified sharp function of $S_{n}^{*} f$ in terms of the maximal function of $f$. We also prove weighted norm inequalities relating the maximal function and sharp functions.

The results in $\S \S 2$ and 3 will be used in the proof of (i) $\Rightarrow$ (ii) of Theorem 1.1. This implication together with the implications (ii) $\Rightarrow$ (i) and (ii) $\Leftrightarrow$ (iii) are proved in $\S 4$.

Theorem 1.2 is proved in $\S 5$, with the implication (i) $\Rightarrow$ (ii) based on a modified Calderón-Zygmund decomposition lemma and Theorem 1.1.

In what follows, $C$ will denote an absolute constant which may vary from line to line.

\section{The Condition $A_{p}(G)$ and the HaRdy-Littlewood maximal fUnCtion}

The following notation will be used throughout this paper. We decompose the collection $\mathscr{I}$ of generalized intervals into disjoint subcollections in the following manner. Let $\mathscr{I}_{-1}=\{G\}$. For $k=0,1, \ldots$, let $\mathscr{I}_{k}$ be the collection of all $I \in \mathscr{I}$ such that $I$ is a proper subset of a coset of $G_{k}$, and is a union of cosets of $G_{k+1}$. The collections $\mathscr{I}_{k}$ are disjoint, and $\mathscr{I}=\bigcup_{k=-1}^{\infty} \mathscr{I}_{k}$.

For $I \in \mathscr{I}$, we define the set $3 I \in \mathscr{I}$ as follows. If $I=G$, let $3 I=G$. For $I \in \mathscr{I}_{k}, k=0,1, \ldots$, there is $x \in G$ such that $I \subset x+G_{k}$. If $\mu(I) \geq$ $\mu\left(G_{k}\right) / 3$, let $3 I=x+G_{k}$. If $\mu(I)<\mu\left(G_{k}\right) / 3$, consider $x+G_{k}$ as a circle. Then $I$ is an interval in this circle. Define $3 I \in \mathscr{I}_{k}$ to be the interval in this circle which contains $I$ at its center and has measure $\mu(3 I)=3 \mu(I)$. In all cases, for $I \in \mathscr{I}, \mu(3 I) \leq 3 \mu(I)$. 
The properties of weights in $A_{p}(G)$ are analogous to the properties of those that satisfy Muckenhoupt's $A_{p}$ condition. (See [1 and 4].)

If $w \in A_{p}(G), 1<p<\infty$, then $w$ and $w^{-1 /(p-1)} \in L^{1}$. Hence $0<w<\infty$ a.e. and $L^{p}(w) \subset L^{1}$. Similarly, if $w \in A_{1}(G)$, then $w \in L^{1}$ and $\operatorname{ess} \inf _{G} w>$ 0 . Hence $0<w<\infty$ a.e. and $L^{1}(w) \subset L^{1}$.

If $w \in A_{p}(G), 1<p<\infty$, then Hölder's inequality implies that there is a constant $C$ such that for any $I \in \mathscr{I}$,

$$
\int_{3 I} w d \mu \leq C \int_{I} w d \mu .
$$

A basic property of $A_{p}(G)$ weights is the reverse Hölder inequality.

Lemma 2.1. Let $w \in A_{p}(G), 1<p<\infty$. Then there exist $s>1$ and $a$ constant $C$ such that for any $I \in \mathcal{I}$,

$$
\left(\frac{1}{\mu(I)} \int_{I} w^{s} d \mu\right)^{1 / s} \leq \frac{C}{\mu(I)} \int_{I} w d \mu .
$$

The following two lemmas are consequences of the reverse Hölder inequality.

Lemma 2.2. Suppose $w \in A_{p}(G), 1<p<\infty$. Then there is $1<q<p$ such that $w \in A_{q}(G)$.

We also observe that if $w \in A_{p}(G), 1 \leq p<\infty$, then $w \in A_{q}(G), p<q<$ $\infty$.

Lemma 2.3. Suppose $w \in A_{p}(G), 1<p<\infty$. Given any $\varepsilon>0$, there is $\delta>0$ such that for any $I \in \mathscr{I}$, and any measurable $E \subset I$ with $\mu(E) \leq \delta \mu(I)$, we have $\int_{E} w d \mu \leq \varepsilon \int_{I} w d \mu$.

The proofs of Lemmas 2.1-2.3 are similar to those given in [1]. (See also [4].)

We now define the Hardy-Littlewood maximal function that is appropriate for the study of Vilenkin-Fourier series. For $f \in L^{1}$, let

$$
M f(x)=\sup _{\substack{x \in I \\ I \in \mathcal{J}}} \frac{1}{\mu(I)} \int_{I}|f| d \mu .
$$

This maximal function was first introduced by P. Simon in [9]. He also showed that the maximal operator is bounded in $L^{p}, 1<p \leq \infty$, and is of weak type $(1,1)$. Using the above properties of $A_{p}(G)$ weights, we obtain the following analogue of Muckenhoupt's theorem [7].

Theorem 2.4. Let $w$ be a weight function on $G$. For $1<p<\infty$, the following two statements are equivalent:

(i) $w \in A_{p}(G)$.

(ii) There is a constant $C$, depending only on $w$ and $p$, such that for every $f \in L^{p}(w)$, we have $f \in L^{1}$ and

$$
\int_{G}(M f)^{p} w d \mu \leq C \int_{G}|f|^{p} w d \mu .
$$

The following two statements are also equivalent: 
(iii) $w \in A_{1}(G)$.

(iv) There is a constant $C$, depending only on $w$, such that for every $f \in$ $L^{1}(w)$, we have $f \in L^{1}$ and

$$
\int_{\{M f>y\}} w d \mu \leq C y^{-1} \int_{G}|f| w d \mu, \quad y>0 .
$$

Proof. The proof of this theorem follows the same lines as those in Muckenhoupt [7] and Coifman and Fefferman [1]. (See also [4].) We shall only discuss one modification which involves the generalized intervals.

To prove that the $A_{p}(G)$ condition is sufficient, suppose $1 \leq p<\infty$ and $w \in A_{p}(G)$. It is enough to prove the weak type inequality

$$
\int_{\{M f>10 y\}} w d \mu \leq C y^{-p} \int_{G}|f|^{p} w d \mu, y>0, \quad f \in L_{p}(w) .
$$

The strong type inequality for $1<p<\infty$ then follows from Lemma 2.2 and the Marcinkiewicz interpolation theorem.

To prove (2.2), we can assume that $y \geq\|f\|_{1}$. (If $y<\|f\|_{1}$, Hölder's inequality and the $A_{p}(G)$ condition imply

$$
\left.\int_{G} w d \mu \leq y^{-p}\|f\|_{1}^{p} \int_{G} w d \mu \leq C y^{-p} \int_{G}|f|^{p} w d \mu .\right)
$$

Apply the decomposition in [10], Lemma 2 to the function $f$ and the value $y$ to obtain a sequence of disjoint generalized intervals $\left\{I_{j}\right\}$ such that

$$
y<\frac{1}{\mu\left(I_{j}\right)} \int_{I_{j}}|f| d \mu \leq 3 y, \quad j=1,2, \ldots,
$$

and

$$
|f(x)| \leq y \quad \text { for a.e. } x \notin \bigcup_{j} I_{j}
$$

We shall show that

$$
\{M f>10 y\} \subset \bigcup_{j}\left(3 I_{j}\right) .
$$

Suppose $x \notin \bigcup_{j}\left(3 I_{j}\right)$. Let $I \in \mathscr{I}$ such that $x \in I$. Then

$$
\begin{aligned}
\int_{I}|f| d \mu & =\int_{I \cap\left(\bigcup_{j} I_{j}\right)}|f| d \mu+\int_{I \cap\left(\bigcup_{j} I_{j}\right)}|f| d \mu \\
& \leq \sum_{j} \int_{I \cap I_{j}}|f| d \mu+y \mu(I),
\end{aligned}
$$

by (2.4). To estimate the second last term, suppose $I \cap I_{j} \neq \varnothing$. From this and the fact that $I \cap^{c}\left(3 I_{j}\right) \neq \varnothing$ (since it contains $x$ ), we have $I_{j} \subset 3 I$. (If $I_{j}$ and $I$ belong to distinct $\mathscr{J}_{k}$ 's, then, since they are not disjoint, one is a subset of the other. From this it follows that $I_{j} \subset I \subset 3 I$. If $I_{j}$ and $I$ belong to the same $\mathscr{I}_{k}$, then they belong to the same coset of $G_{k}$, and we obtain $I_{j} \subset 3 I$ from a geometric observation.) Hence, by (2.3),

$$
\begin{aligned}
\sum_{j} \int_{I \cap I_{j}}|f| d \mu & \leq \sum_{I_{j} \subset 3 I} \int_{I_{j}}|f| d \mu \leq \sum_{I_{j} \subset 3 I} 3 y \mu\left(I_{j}\right) \\
& \leq 3 y \mu(3 I) \leq 9 y \mu(I) .
\end{aligned}
$$


Therefore, for all such $I, \int_{I}|f| d \mu \leq 10 y \mu(I)$, and we have $\{M f>10 y\} \subset$ $\bigcup_{j}\left(3 I_{j}\right)$.

The rest of the proof of $(2.2)$ then follows as in the above references, using (2.1), (2.3) and the $A_{p}(G)$ condition. This concludes the proof of Theorem 2.4.

\section{THE SHARP FUNCTIONS}

In this section, we introduce a sequence of sharp functions $\left\{f_{n}^{\#}\right\}$, which will be used to prove (i) $\Rightarrow$ (ii) of Theorem 1.1. Our sharp functions are modifications of that in Fefferman and Stein [3]. Before we define these modified sharp functions, we first introduce the functions $f_{I, n}$ which take the place of the mean value of $f$ over $I$ in the original sharp function.

Let $n$ be a nonnegative integer expressed in the form $n=\sum_{k=0}^{\infty} \alpha_{k} m_{k}$, $0 \leq \alpha_{k}<p_{k}$. For $I \in \mathscr{I}$ and $f \in L^{1}$, define $f_{I, n}$ as follows. If $I=G$, let $f_{I, n}=\int_{G} f d \mu$. For $I \in \mathscr{I}_{k}, k=0,1, \ldots$, let $f_{I, n}$ be a function of the form $f_{I, n}(x)=a_{I, n}(f)+b_{I, n}(f) \phi_{k}^{-\alpha_{k}}(x)$, where $a_{I, n}(f)$ and $b_{I, n}(f)$ are constants, such that

$$
\int_{I} f d \mu=\int_{I} f_{I, n} d \mu
$$

and

$$
\int_{I} f \phi_{k}^{\alpha_{k}} d \mu=\int_{I} f_{I, n} \phi_{k}^{\alpha_{k}} d \mu .
$$

If $I$ is not a coset of $G_{k+1}$, and if $\alpha_{k} \neq 0$, then $\left|(\mu(I))^{-1} \int_{I} \phi_{k}^{\alpha_{k}} d \mu\right| \neq 1$, and the choice for $a_{I, n}(f)$ and $b_{I, n}(f)$ is unique. (See [10, pp. 315-316].) If $I$ is a coset of $G_{k+1}$, or if $\alpha_{k}=0$, then $\phi_{k}^{-\alpha_{k}}(x)$ is a constant in $I$. In this case, we define $a_{I, n}(f)=(\mu(I))^{-1} \int_{I} f d \mu$, and $b_{I, n}(f)=0$. For $n=0,1, \ldots$, $f \in L^{1}$, define the $n$th sharp function as

$$
f_{n}^{\#}(x)=\sup _{\substack{x \in I \\ I \in I}} \frac{1}{\mu(I)} \int_{I}\left|f-f_{I, n}\right| d \mu .
$$

The functions $f_{I, n}$ and $f_{n}^{\#}$ have the following properties.

Lemma 3.1. There is a constant $C$ such that for any $f \in L^{1}, I \in \mathscr{I}, n=$ $0,1, \ldots$, we have

$$
\left|f_{I, n}(x)\right| \leq \frac{C}{\mu(I)} \int_{I}|f| d \mu, \quad x \in I .
$$

Proof. The proof is the same as that for (25) in [10]. See [10, pp. 315-316].

In the following, we shall set $\alpha_{-1}=0$ for the sake of convenience.

Lemma 3.2. There is a constant $C$ such that for any constants $A$ and $B$, we have

$$
\int_{I}\left|f-f_{I, n}\right| d \mu \leq C \int_{I}\left|f-A-B \phi_{k}^{-\alpha_{k}}\right| d \mu,
$$


for all $f \in L^{1}, n=\sum_{k=0}^{\infty} \alpha_{k} m_{k}, 0 \leq \alpha_{k}<p_{k}$, and $I \in \mathscr{I}_{k}, k=-1,0,1, \ldots$. Proof. We observe that if $I$ is a coset of $G_{k}, k=0,1, \ldots$, or if $I \in \mathscr{I}_{k}$, $k=0,1, \ldots$ and $\alpha_{k}=0$, then $\phi_{k}^{-\alpha_{k}}(x)$ is constant in $I$. In these cases, we may assume $B=0$. (3.3) holds with $C=2$ since $f_{I, n}=(\mu(I))^{-1} \int_{I} f d \mu$. Then

Now, suppose $I \in \mathscr{I}_{k}, k=0,1, \ldots, I$ is not a coset of $G_{k+1}$, and $\alpha_{k} \neq 0$.

$$
\begin{aligned}
\int_{I}\left|f-f_{I, n}\right| d \mu \leq & \int_{I}\left|f-A-B \phi_{k}^{-\alpha_{k}}\right| d \mu \\
& +\int_{I}\left|\left(a_{I, n}(f)-A\right)+\left(b_{I, n}(f)-B\right) \phi_{k}^{-\alpha_{k}}\right| d \mu .
\end{aligned}
$$

By (3.1) and (3.2),

$$
\int_{I}\left(f-A-B \phi_{k}^{-\alpha_{k}}\right) d \mu=\int_{I}\left\{\left(a_{I, n}(f)-A\right)+\left(b_{I, n}(f)-B\right) \phi_{k}^{-\alpha_{k}}\right\} d \mu
$$

and

$$
\int_{I}\left(f-A-B \phi_{k}^{-\alpha_{k}}\right) d \mu=\int_{I}\left\{\left(a_{I, n}(f)-A\right)+\left(b_{I, n}(f)-B\right) \phi_{k}^{-\alpha_{k}}\right\} \phi_{k}^{\alpha_{k}} d \mu .
$$

Therefore, by the uniqueness of the coefficients, we have

$$
\left(f-A-B \phi_{k}^{-\alpha_{k}}\right)_{I, n}=\left(a_{I, n}(f)-A\right)+\left(b_{I, n}(f)-B\right) \phi_{k}^{-\alpha_{k}} .
$$

It then follows from Lemma 3.1 that

$$
\begin{gathered}
\int_{I}\left|\left(a_{I, n}(f)-A\right)+\left(b_{I, n}(f)-B\right) \phi_{k}^{-\alpha_{k}}\right| d \mu \\
\quad=\int_{I}\left|\left(f-A-B \phi_{k}^{-\alpha_{k}}\right)_{I, n}\right| d \mu \\
\leq C \int_{I}\left|f-A-B \phi_{k}^{-\alpha_{k}}\right| d \mu .
\end{gathered}
$$

This proves Lemma 3.2.

For $f \in L^{r}, 1<r<\infty$, let

$$
M_{r} f(x)=\sup _{\substack{x \in I \\ I \in \mathcal{I}}}\left(\frac{1}{\mu(I)} \int_{I}|f|^{r} d \mu\right)^{1 / r} .
$$

We have the following pointwise estimate concerning the $n$th sharp function of the $n$th modified partial sum $S_{n}^{*} f$.

Theorem 3.3. Let $1<r<\infty$. There is a constant $C$, depending only on $r$, such that for any $f \in L^{r}, n=1,2, \ldots$,

$$
\left(S_{n}^{*} f\right)_{n}^{\#}(x) \leq C M_{r} f(x), \quad x \in G .
$$

Proof: Let $x \in G$, and $n=\sum_{k=0}^{\infty} \alpha_{k} m_{k}, 0 \leq \alpha_{k}<p_{k}$. From Lemma 3.2, it is sufficient to show that for any $I \in \mathscr{I}_{k}, k=-1,0,1, \ldots$, with $x \in I$, there exist constants $A$ and $B$ (depending on $I, n$ and $f$ ) such that 


$$
\frac{1}{\mu(I)} \int_{I}\left|S_{n}^{*} f-A-B \phi_{k}^{-\alpha_{k}}\right| d \mu \leq C M_{r} f(x),
$$

where $C$ depends only on $r$.

Suppose $I \in \mathscr{I}_{k}, k=-1,0,1, \ldots$, with $x \in I$. Let $f=f_{1}+f_{2}$, where $f_{1}=f \chi_{3 I}$. Then $S_{n}^{*} f=S_{n}^{*} f_{1}+S_{n}^{*} f_{2}$. By Theorem $1^{*}$ of [10], we have

$$
\begin{aligned}
\frac{1}{\mu(I)} \int_{I}\left|S_{n}^{*} f_{1}\right| d \mu & \leq\left(\frac{1}{\mu(I)} \int_{G}\left|S_{n}^{*} f_{1}\right|^{r} d \mu\right)^{1 / r} \leq C\left(\frac{1}{\mu(I)} \int_{G}\left|f_{1}\right|^{r} d \mu\right)^{1 / r} \\
& \leq C\left(\frac{1}{\mu(3 I)} \int_{3 I}|f|^{r} d \mu\right)^{1 / r} \leq C M_{r} f(x)
\end{aligned}
$$

For $I=G, f_{2}=0$, and (3.4) follows with $A=B=0$. From now on, we can assume $k \geq 0$.

To estimate $S_{n}^{*} f_{2}$, let $c$ be a fixed point in $I$, and $y \in I$. By (1.6) we have

$$
S_{n}^{*} f_{2}(y)-S_{n}^{*} f_{2}(c)=\sum_{l=0}^{\infty}\left\{S_{\alpha_{l} m_{l}}^{*} f_{2}(y)-S_{\alpha_{l} m_{l}}^{*} f_{2}(c)\right\}
$$

Expand each term on the right according to (1.7). For $l \geq k+1, y+G_{l}$ and $c+G_{l}$ are subsets of $I$. Therefore $f_{2}$ vanishes in these cosets. For $0 \leq l \leq k$, $y+G_{l}=c+G_{l}$. Hence

$$
\begin{aligned}
S_{n}^{*} f_{2}(y)-S_{n}^{*} f_{2}(c) & \\
=\sum_{l=0}^{k} \frac{1}{\mu\left(G_{l}\right)} \int_{y+G_{l}} f_{2}(t)\left\{\phi_{l}^{-\alpha_{l}}(y-t)\right. & \left(\sum_{j=0}^{\alpha_{l}-1} \phi_{l}^{j}(y-t)\right) \\
& \left.-\phi_{l}^{-\alpha_{l}}(c-t)\left(\sum_{j=0}^{\alpha_{l}-1} \phi_{l}^{j}(c-t)\right)\right\} d \mu(t) .
\end{aligned}
$$

Now, for any $t \in G, y-t$ and $c-t$ belong to the same coset of $G_{l}$, for any $0 \leq l \leq k$. Since $\phi_{l}$ is constant on each coset of $G_{l+1}, l=0,1, \ldots$, we have $\phi_{l}(y-t)=\phi_{l}(c-t), 0 \leq l \leq k-1$. Therefore all integrals on the right vanish except for the term with $l=k$. We thus have

$$
S_{n}^{*} f_{2}(y)-S_{n}^{*} f_{2}(c)=S_{\alpha_{k} m_{k}}^{*} f_{2}(y)-S_{\alpha_{k} m_{k}}^{*} f_{2}(c) .
$$

The constants $A$ and $B$ are defined in terms of the conjugate functions (see (1.8)). Let $A=S_{n}^{*} f_{2}(c)-i \phi_{k}^{-\alpha_{k}}(c) H_{k}\left(f_{2} \phi_{k}^{\alpha_{k}}\right)(c)$ and $B=i H_{k}\left(f_{2} \phi_{k}^{\alpha_{k}}\right)(c)$. For $y \in I$, it follows from (3.6) that

$$
\begin{aligned}
S_{n}^{*} f_{2}(y)-A-B \phi_{k}^{-\alpha_{k}}(y)= & S_{\alpha_{k} m_{k}}^{*} f_{2}(y)-S_{\alpha_{k} m_{k}}^{*} f_{2}(c) \\
& +i \phi_{k}^{-\alpha_{k}}(c) H_{k}\left(f_{2} \phi_{k}^{\alpha_{k}}\right)(c)-i \phi_{k}^{-\alpha_{k}}(y) H_{k}\left(f_{2} \phi_{k}^{\alpha_{k}}\right)(c) .
\end{aligned}
$$


Using (1.9), we express the right side as a sum of the following terms:

$$
\begin{aligned}
E_{1}= & \frac{\alpha_{k}}{\mu\left(G_{k}\right)} \int_{\left(y+G_{k}\right) \cap\left\{y_{k}=t_{k}\right\}} f_{2}(t) d \mu(t) \\
& -\frac{\alpha_{k}}{\mu\left(G_{k}\right)} \int_{\left(c+G_{k}\right) \cap\left\{c_{k}=t_{k}\right\}} f_{2}(t) d \mu(t), \\
E_{2}= & \frac{1}{2} \phi_{k}^{-\alpha_{k}}(y) \frac{1}{\mu\left(G_{k}\right)} \int_{\left(y+G_{k}\right) \cap\left\{y_{k} \neq t_{k}\right\}} f_{2}(t) \phi_{k}^{\alpha_{k}}(t) d \mu(t) \\
& -\frac{1}{2} \frac{1}{\mu\left(G_{k}\right)} \int_{\left(y+G_{k}\right) \cap\left\{y_{k} \neq t_{k}\right\}} f_{2}(t) d \mu(t) \\
& -\frac{1}{2} \phi_{k}^{-\alpha_{k}}(c) \frac{1}{\mu\left(G_{k}\right)} \int_{\left(c+G_{k}\right) \cap\left\{c_{k} \neq t_{k}\right\}} f_{2}(t) \phi_{k}^{\alpha_{k}}(t) d \mu(t) \\
& +\frac{1}{2} \frac{1}{\mu\left(G_{k}\right)} \int_{\left(c+G_{k}\right) \cap\left\{c_{k} \neq t_{k}\right\}} f_{2}(t) d \mu(t), \\
E_{3}= & i \phi_{k}^{-\alpha_{k}}(y)\left\{H_{k}\left(f_{2} \phi_{k}^{\alpha_{k}}\right)(y)-H_{k}\left(f_{2} \phi_{k}^{\alpha_{k}}\right)(c)\right\}, \\
E_{4}= & -i\left\{H_{k} f_{2}(y)-H_{k} f_{2}(c)\right\} .
\end{aligned}
$$

We shall show that $E_{1}=0$, and each of $\left|E_{2}\right|,\left|E_{3}\right|$ and $\left|E_{4}\right|$ is dominated by $C M f(x)$, and hence by $C M_{r} f(x)$. (3.4) will then follow from (3.5), (3.7), (3.8) and these estimates.

Since $f_{2}$ vanishes in $I$, and hence in $\left(y+G_{k}\right) \cap\left\{y_{k}=t_{k}\right\}$ and $\left(c+G_{k}\right) \cap$ $\left\{c_{k}=t_{k}\right\}$, we have $E_{1}=0$.

Next, the absolute value of each of the terms in $E_{2}$ is bounded by

$$
\left(2 \mu\left(G_{k}\right)\right)^{-1} \int_{x+G_{k}}|f| d \mu .
$$

Therefore $\left|E_{2}\right| \leq 2 M f(x)$.

To estimate $E_{3}$, we observe that for $y \in I$,

$$
H_{k}\left(f_{2} \phi_{k}^{\alpha_{k}}\right)(y)=\frac{1}{2} \frac{1}{\mu\left(G_{k}\right)} \int_{\left(x+G_{k}\right) \cap c(3 I)} f(t) \phi_{k}^{\alpha_{k}}(t) \cot \left(\pi\left(y_{k}-t_{k}\right) / p_{k}\right) d \mu(t),
$$

since $f_{2}=0$ in $3 I$ and $\left(y+G_{k}\right) \cap\left\{y_{k} \neq t_{k}\right\} \supset\left(x+G_{k}\right) \cap^{c}(3 I)$. A similar expression holds for $H_{k}\left(f_{2} \phi_{k}^{\alpha_{k}}\right)(c)$. Hence

$$
\begin{aligned}
\left|E_{3}\right| & =\left|H_{k}\left(f_{2} \phi_{k}^{\alpha_{k}}\right)(y)-H_{k}\left(f_{2} \phi_{k}^{\alpha_{k}}\right)(c)\right| \\
& \leq \frac{1}{2} \frac{1}{\mu\left(G_{k}\right)} \int_{\left(x+G_{k}\right) \cap c(3 I)}|f(t)| \mid \cot \left(\frac{\pi\left(y_{k}-t_{k}\right)}{p_{k}}\right) \\
& -\cot \left(\frac{\pi\left(c_{k}-t_{k}\right)}{p_{k}}\right) \mid d \mu(t) .
\end{aligned}
$$

Let $3^{j+1} I=3\left(3^{j} I\right), j=1,2, \ldots$ If $3 I \neq x+G_{k}$, write $\left(x+G_{k}\right) \cap^{c}(3 I)=$ $\bigcup_{j=1}^{J-1}\left(x+G_{k}\right) \cap\left(3^{j+1} I \backslash 3^{j} I\right)$, where $J=\min \left\{j \geq 1: 3^{j} I=x+G_{k}\right\}$. Now, for $1 \leq j \leq J-1$ and $t \in\left(x+G_{k}\right) \cap\left(3^{j+1} I \backslash 3^{j} I\right)$,

$$
\begin{aligned}
& \left|\cot \left(\frac{\pi\left(y_{k}-t_{k}\right)}{p_{k}}\right)-\cot \left(\frac{\pi\left(c_{k}-t_{k}\right)}{p_{k}}\right)\right| \\
& \quad=\left|\sin \left(\frac{\pi\left(y_{k}-c_{k}\right)}{p_{k}}\right) /\left\{\sin \left(\frac{\pi\left(y_{k}-t_{k}\right)}{p_{k}}\right) \sin \left(\frac{\pi\left(c_{k}-t_{k}\right)}{p_{k}}\right)\right\}\right| \\
& \quad \leq C \mu(I) \mu\left(G_{k}\right) /\left(\mu\left(3^{j-1} I\right)\right)^{2} \leq C 3^{-j} \mu\left(G_{k}\right) / \mu\left(3^{j+1} I\right) .
\end{aligned}
$$


Substituting these into (3.9), we obtain

$$
\begin{aligned}
\left|E_{3}\right| & \leq C \sum_{j=1}^{J-1} 3^{-j} \frac{1}{\mu\left(3^{j+1} I\right)} \int_{3^{j+1} I}|f(t)| d \mu(t) \\
& \leq C \sum_{j=1}^{J-1} 3^{-j} M f(x) \leq C M f(x) .
\end{aligned}
$$

The same argument shows that $\left|E_{4}\right| \leq C M f(x)$. This completes the proof of Theorem 3.3.

We have the following weighted norm inequality relating the maximal function and the sharp functions.

Theorem 3.4. Let $1<p<\infty, w \in A_{p}(G)$. There is a constant $C$, depending only on $p$ and $w$, such that for any $f \in L^{p}(w), n=0,1, \ldots$,

$$
\int_{G}(M f)^{p} w d \mu \leq C \int_{G}\left(f_{n}^{\#}\right)^{p} w d \mu+C\left(\int_{G}|f| d \mu\right)^{p} \int_{G} w d \mu .
$$

Proof. Let $f \in L^{p}(w)$. We observe that $w \in A_{p}(G)$ implies that $f \in L^{1}$. Let $y_{0}=\|f\|_{1}$ and $n$ be a nonnegative integer. For any $y>y_{0}$, apply the decomposition in [10, Lemma 2] to the function $f$ and the value $y$ to obtain a sequence of disjoint generalized intervals $\left\{I_{j}\right\}_{j \geq 1}$ such that

$$
y<\frac{1}{\mu\left(I_{j}\right)} \int_{I_{j}}|f| d \mu \leq 3 y, \quad j=1,2, \ldots,
$$

and

$$
|f(x)| \leq y \quad \text { for a.e. } x \notin \bigcup_{j} I_{j} .
$$

From Lemma 3.1, there is a constant $A>1$, independent of $f$, such that for any $I \in \mathscr{I}, n=0,1, \ldots$,

$$
\left|f_{I, n}(x)\right| \leq \frac{A}{\mu(I)} \int_{I}|f| d \mu, \quad x \in I .
$$

Since $y<4 A y$, the above decomposition applied to $f$ and the value $4 A y$ gives a sequence of disjoint generalized intervals $\left\{I_{i j}\right\}_{i, j \geq 1}$ such that $\bigcup_{i} I_{i j} \subset I_{j}$, $j=1,2, \ldots$,

$$
4 A y<\frac{1}{\mu\left(I_{i j}\right)} \int_{I_{i j}}|f| d \mu \leq 12 A y, \quad i, j=1,2, \ldots,
$$

and

$$
|f(x)| \leq 4 A y \quad \text { for a.e. } x \notin \bigcup_{i, j} I_{i j} .
$$

Let $\delta>0$ be a constant whose value is to be chosen later. For each $I_{j}$, there are two possibilities.

Case 1. $I_{j} \subset\left\{f_{n}^{\#}>\delta A y\right\}$. Then

$$
\sum_{i} \int_{I_{i j}} w d \mu \leq \int_{\left\{f_{n}^{*}>\delta A y\right\} \cap I_{j}} w d \mu
$$


Case 2. $I_{j} \not \subset\left\{f_{n}^{\#}>\delta A y\right\}$. Then

$$
\frac{1}{\mu\left(I_{j}\right)} \int_{I_{j}}\left|f-f_{I_{j}, n}\right| d \mu \leq \delta A y .
$$

Hence

$$
\delta A y \mu\left(I_{j}\right) \geq \sum_{i}\left(\int_{I_{i j}}|f| d \mu-\int_{I_{i j}}\left|f_{I_{j}, n}\right| d \mu\right) .
$$

By (3.13), we have

$$
\int_{I_{i j}}|f| d \mu \geq 4 A y \mu\left(I_{i j}\right) .
$$

Also, from (3.12) and (3.11), we have, for $x \in I_{i j} \subset I_{j}$,

$$
\left|f_{I_{j, n}}(x)\right| \leq A\left(\mu\left(I_{j}\right)\right)^{-1} \int_{I_{j}}|f| d \mu \leq 3 A y .
$$

Hence

$$
\int_{I_{i j}}\left|f_{I_{j}, n}\right| d \mu \leq 3 A y \mu\left(I_{i j}\right)
$$

Substituting these estimates into (3.14), we obtain

$$
\mu\left(\bigcup_{i} I_{i j}\right) \leq \delta \mu\left(I_{j}\right)
$$

For $\varepsilon>0$ to be chosen later, let $\delta$ be the value that associates with $\varepsilon$ in Lemma 2.3. Then

$$
\sum_{i} \int_{I_{i j}} w d \mu \leq \varepsilon \int_{I_{j}} w d \mu .
$$

Summing over all $I_{j}$ in Cases 1 and 2, we obtain

$$
\sum_{i, j} \int_{I_{i j}} w d \mu \leq \int_{\left\{f_{n}^{*}>\delta A y\right\}} w d \mu+\varepsilon \int_{\bigcup_{j} I_{j}} w d \mu .
$$

Now, from (2.5), we have $\{M f>40 A y\} \subset \bigcup_{i, j}\left(3 I_{i j}\right)$. Hence, by (2.1), there is a constant $B$ such that

$$
\int_{\{M f>40 A y\}} w d \mu \leq B \sum_{i, j} \int_{I_{i j}} w d \mu .
$$

Also, $\bigcup_{j} I_{j} \subset\{M f>y\}$. Therefore, for $y \geq y_{0}$, we have

$$
\int_{\{M f>40 A y\}} w d \mu \leq B \int_{\left\{f_{n}^{*}>\delta A y\right\}} w d \mu+B \varepsilon \int_{\{M f>y\}} w d \mu .
$$

Multiply both sides of the above inequality by $p y^{p-1}$ and integrate over $y \in$ $\left[y_{0}, \infty\right)$. We obtain

$$
\begin{aligned}
& p \int_{y_{0}}^{\infty} y^{p-1} \int_{\{M f>40 A y\}} w d \mu d y \\
& \quad \leq B(\delta A)^{-p} \int_{G}\left(f_{n}^{\#}\right)^{p} w d \mu+B \varepsilon \int_{G}(M f)^{p} w d \mu
\end{aligned}
$$


Also,

$$
p \int_{0}^{y_{0}} y^{p-1} \int_{\{M f>40 A y\}} w d \mu d y \leq\left(\int_{G}|f| d \mu\right)^{p} \int_{G} w d \mu
$$

Therefore, we have

$$
\begin{aligned}
\int_{G}(M f)^{p} w d \mu \leq & B(40 / \delta)^{p} \int_{G}\left(f_{n}^{\#}\right)^{p} w d \mu \\
& +B \varepsilon(40 A)^{p} \int_{G}(M f)^{p} w d \mu \\
& +(40 A)^{p}\left(\int_{G}|f| d \mu\right)^{p} \int_{G} w d \mu
\end{aligned}
$$

Since $f \in L^{p}(w)$ and $w \in A_{p}(G)$, we have $M f \in L^{p}(w)$, by Theorem 2.4. Choosing $\varepsilon=(2 B)^{-1}(40 A)^{-p}$, we obtain (3.10). This completes the proof of Theorem 3.4.

\section{Proof of Theorem 1.1}

We shall prove Theorem 1.1 by showing (i) $\Leftrightarrow$ (ii) $\Leftrightarrow$ (iii) .

Proof of $(\mathrm{i}) \Rightarrow$ (ii). Suppose $w \in A_{p}(G)$. We have $L^{p}(w) \subset L^{1}$. It is sufficient to prove (1.2) with $S_{n}$ replaced by $S_{n}^{*}$. Let $f \in L^{p}(w)$. We observe that $S_{n}^{*} f \in L^{p}(w)$, since for $x \in G$,

$$
\left|S_{n}^{*} f(x)\right| \leq n \int_{G}|f| d \mu \leq n\left(\int_{G}|f|^{p} w d \mu\right)^{1 / p}\left(\int_{G} w^{-1 /(p-1)} d \mu\right)^{1-1 / p},
$$

by Hölder's inequality. Therefore, using the $A_{p}(G)$ condition, we have

$$
\int_{G}\left|S_{n}^{*} f\right|^{p} w d \mu \leq C n^{p} \int_{G}|f|^{p} w d \mu<\infty .
$$

We now apply Theorem 3.4 to the function $S_{n}^{*} f$ to get

$$
\begin{aligned}
\int_{G}\left|S_{n}^{*} f\right|^{p} w d \mu & \leq \int_{G}\left(M\left(S_{n}^{*} f\right)\right)^{p} w d \mu \\
& \leq C \int_{G}\left(\left(S_{n}^{*} f\right)_{n}^{\#}\right)^{p} w d \mu+C\left(\int_{G}\left|S_{n}^{*} f\right| d \mu\right)^{p} \int_{G} w d \mu .
\end{aligned}
$$

Since $w \in A_{p}(G)$, Lemma 2.2 shows there is $1<r<p$ such that $w \in$ $A_{p / r}(G)$. Hence $|f|^{r} \in L^{p / r}(w) \subset L^{1}$, and it follows from Theorem 3.3 and Theorem 2.4 that

$$
\begin{aligned}
\int_{G}\left(\left(S_{n}^{*} f\right)_{n}^{\#}\right)^{p} w d \mu & \leq C \int_{G}\left(M_{r} f\right)^{p} w d \mu \\
& =C \int_{G}\left(M|f|^{r}\right)^{p / r} w d \mu \leq C \int_{G}|f|^{p} w d \mu .
\end{aligned}
$$

Also, by Theorem $1^{*}$ of [10],

$$
\int_{G}\left|S_{n}^{*} f\right| d \mu \leq\left(\int_{G}\left|S_{n}^{*} f\right|^{r} d \mu\right)^{1 / r} \leq C\left(\int_{G}|f|^{r} d \mu\right)^{1 / r} .
$$


Therefore, Hölder's inequality gives

$$
\begin{aligned}
& \left(\int_{G}\left|S_{n}^{*} f\right| d \mu\right)^{p} \int_{G} w d \mu \\
& \quad \leq C\left(\int_{G}|f|^{p} w d \mu\right)\left(\int_{G} w^{-1 /(p / r-1)} d \mu\right)^{p / r-1}\left(\int_{G} w d \mu\right) \\
& \leq C \int_{G}|f|^{p} w d \mu,
\end{aligned}
$$

since $w \in A_{p / r}(G)$. Substituting (4.2) and (4.3) into (4.1), we obtain

$$
\int_{G}\left|S_{n}^{*} f\right|^{p} w d \mu \leq C \int_{G}|f|^{p} w d \mu .
$$

This completes the proof of (i) $\Rightarrow$ (ii) .

Proof of (ii) $\Rightarrow$ (i). Suppose $w$ satisfies (ii). As a consequence of $L^{p}(w) \subset L^{1}$, we have $w^{-1 /(p-1)} \in L^{1}$. (Otherwise, $w^{-1 / p} \notin L^{p^{\prime}}$, where $1 / p+1 / p^{\prime}=1$. Hence there exists $g \in L^{p}$ such that $g w^{-1 / p} \notin L^{1}$, contradicting $g w^{-1 / p} \in$ $L^{p}(w)$.) Therefore $\left\|w^{-1 /(p-1)}\right\|_{p, w}^{p}=\left\|w^{-1 /(p-1)}\right\|_{1}<\infty$.

To prove (1.1) for $I=G$, let $f=w^{-1 /(p-1)}$. Then, for $x \in G, S_{1} f(x)=$ $\|f\|_{1}=\left\|w^{-1 /(p-1)}\right\|_{1}>0$, otherwise we have $w \equiv \infty$ a.e. Hence, for every $0<y \leq\|f\|_{1}$, it follows from (1.2) that

$$
\int_{G} w d \mu=\int_{\left\{S_{1} f>y\right\}} w d \mu \leq C y^{-p} \int_{G}|f|^{p} w d \mu .
$$

Thus

$$
\int_{G} w d \mu \leq C\left(\int_{G} f d \mu\right)^{-p} \int_{G}|f|^{p} w d u,
$$

and hence

$$
\left(\int_{G} w d \mu\right)\left(\int_{G} w^{-1 /(p-1)} d \mu\right)^{p-1} \leq C .
$$

Next, consider $I \in \mathscr{I}_{k}, k=0,1, \ldots$ We can assume $\int_{I} w^{-1 /(p-1)} d \mu>0$, or else there is nothing to prove. First let us assume $\mu(I) \leq \mu\left(G_{k}\right) / 2$. Take $\alpha_{k}=\left[\mu\left(G_{k}\right) /(2 \mu(I))\right]$, where $[a]$ is the largest integer less than or equal to $a$. We have $\alpha_{k} \geq 1$. Let $f=w^{-1 /(p-1)} \chi_{I}$. From (1.7), we have, for $x \in G$,

$$
\begin{aligned}
S_{\alpha_{k} m_{k}} f(x) & =\frac{1}{\mu\left(G_{k}\right)} \int_{I} f(t)\left(\sum_{j=0}^{\alpha_{k}-1} \phi_{k}^{j}(x-t)\right) d \mu(t) \\
& =\frac{1}{\mu\left(G_{k}\right)} \int_{I} f(t) \phi_{k}^{\left(\alpha_{k}-1\right) / 2}(x-t) \frac{\sin \left(\pi \alpha_{k}\left(x_{k}-t_{k}\right) / p_{k}\right)}{\sin \left(\pi\left(x_{k}-t_{k}\right) / p_{k}\right)} d \mu(t) .
\end{aligned}
$$

Hence

$$
\begin{aligned}
& \phi_{k}^{-\left(\alpha_{k}-1\right) / 2}(x) S_{\alpha_{k} m_{k}}\left(f \phi_{k}^{\left(\alpha_{k}-1\right) / 2}\right)(x) \\
& \quad=\frac{1}{\mu\left(G_{k}\right)} \int_{I} f(t) \frac{\sin \left(\pi \alpha_{k}\left(x_{k}-t_{k}\right) / p_{k}\right)}{\sin \left(\pi\left(x_{k}-t_{k}\right) / p_{k}\right)} d \mu(t) .
\end{aligned}
$$

Since for $x, t \in I$,

$$
\frac{\sin \left(\pi \alpha_{k}\left(x_{k}-t_{k}\right) / p_{k}\right)}{\sin \left(\pi\left(x_{k}-t_{k}\right) / p_{k}\right)} \geq \frac{2 \alpha_{k}}{\pi} \geq \frac{\mu\left(G_{k}\right)}{2 \pi \mu(I)},
$$


we have, for $x \in I$,

$$
\phi_{k}^{-\left(\alpha_{k}-1\right) / 2}(x) S_{\alpha_{k} m_{k}}\left(f \phi_{k}^{\left(\alpha_{k}-1\right) / 2}\right)(x) \geq \frac{1}{2 \pi \mu(I)} \int_{I} f(t) d \mu(t) .
$$

Therefore, for any $0<y<(2 \pi \mu(I))^{-1} \int_{I} f d \mu$,

$$
I \subset\left\{\phi_{k}^{-\left(\alpha_{k}-1\right) / 2} S_{\alpha_{k} m_{k}}\left(f \phi_{k}^{\left(\alpha_{k}-1\right) / 2}\right)>y\right\} .
$$

Thus, by (1.2),

$$
\begin{gathered}
\int_{I} w d \mu \leq \int_{\left\{\left|S_{\alpha_{k} m_{k}}\left(f \phi_{k}^{\left(\alpha_{k}-1\right) / 2}\right)\right|>y\right\}} w d \mu \\
\leq C y^{-p} \int_{G}\left|f \phi_{k}^{\left(\alpha_{k}-1\right) / 2}\right|^{p} w d \mu \\
=C y^{-p} \int_{I}|f|^{p} w d \mu .
\end{gathered}
$$

It follows that

$$
\int_{I} w d \mu \leq C(2 \pi \mu(I))^{p}\left(\int_{I} f d \mu\right)^{-p} \int_{I}|f|^{p} w d \mu,
$$

and hence

$$
\left(\int_{I} w d \mu\right)\left(\int_{I} w^{-1 /(p-1)} d \mu\right)^{p-1} \leq C(\mu(I))^{p} .
$$

We have thus proved (1.1) for $I=G$, and for $I \in \mathscr{I}_{k}, k=0,1, \ldots$, with $\mu(I) \leq \mu\left(G_{k}\right) / 2$. In particular, since for $k=1,2, \ldots$, every coset of $G_{k}$ is in $\mathscr{I}_{k-1}$ and $\mu\left(G_{k}\right) \leq \mu\left(G_{k-1}\right) / 2,(1.1)$ holds for all cosets of $G_{k}, k=0,1, \ldots$.

For the case $I \in \mathscr{I}_{k}, k=0,1, \ldots, \mu(I)>\mu\left(G_{k}\right) / 2$, assume $I \subset x+G_{k}$, $x \in G$. Then

$$
\begin{aligned}
& \left(\int_{I} w d \mu\right)\left(\int_{I} w^{-1 /(p-1)} d \mu\right)^{p-1} \\
& \quad \leq\left(\int_{x+G_{k}} w d \mu\right)\left(\int_{x+G_{k}} w^{-1 /(p-1)} d \mu\right)^{p-1} \\
& \quad \leq C\left(\mu\left(G_{k}\right)\right)^{p} \leq C 2^{p}(\mu(I))^{p} .
\end{aligned}
$$

This completes the proof of (ii) $\Rightarrow(\mathrm{i})$.

Before we prove (ii) $\Leftrightarrow$ (iii), we introduce the following notation. For $k=$ $0,1, \ldots$, let $\mathscr{F}_{k}$ be the $\sigma$-algebra generated by the cosets of $G_{k}$. We also say that a function $f$ belongs to $\mathscr{F}_{k}$, or $f \in \mathscr{F}_{k}$, if $f$ is measurable with respect to $\mathscr{F}_{k}$.

Proof of (ii) $\Rightarrow$ (iii). Let $f \in L^{p}(w)$. Since (ii) $\Rightarrow(\mathrm{i})$, we have $w \in L^{1}$. Therefore, given $\varepsilon>0$, there is $g \in \mathscr{F}_{k}$ for some $k=0,1, \ldots$, such that $\|f-g\|_{p, w}<\varepsilon$. Now, for $n \geq m_{k}, S_{n} g=g$. Hence, for all such $n$,

$$
\begin{aligned}
\left\|S_{n} f-f\right\|_{p, w} & \leq\left\|S_{n}(f-g)\right\|_{p, w}+\left\|S_{n} g-g\right\|_{p, w}+\|g-f\|_{p, w} \\
& \leq C\|f-g\|_{p, w}<C \varepsilon,
\end{aligned}
$$

by (1.2). This proves (1.3). 
Proof of (iii) $\Rightarrow$ (ii). We first show that $w \in L^{1}$. Let $f \in L^{p}(w)$ such that $f \notin$ $\mathscr{F}_{k}$ for all $k=0,1, \ldots$ ( $f$ can be obtained as follows. If $w \notin \mathscr{F}_{k}$ for any $k=0,1, \ldots$, take $f=w^{-1 / p}$. Otherwise $w \in \mathscr{F}_{k}$ for some $k=0,1, \ldots$ Since $w \not \equiv \infty$ a.e., there is a coset $I$ of $G_{k}$ such that $w(x)=c<\infty$ for $x \in I$. Let $f$ be any function not in $\mathscr{F}_{k}$ for all $k=0,1, \ldots, f=0$ outside $I$ and $\|f\|_{p}<\infty$.) For this $f, \hat{f}(n)=\int_{G} f \bar{\chi}_{n} d \mu \neq 0$ for infinitely many $n$. But $|\hat{f}(n)|\|w\|_{1}^{1 / p}=\left\|\hat{f}(n) \chi_{n}\right\|_{p, w}=\left\|S_{n+1} f-S_{n} f\right\|_{p, w} \rightarrow 0$ as $n \rightarrow \infty$ by (1.3). This shows $w \in L^{1}$.

From the proof of (ii) $\Rightarrow(\mathrm{i})$, we know that $w$ satisfies $L^{p}(w) \subset L^{1}$ implies that $w^{-1 /(p-1)} \in L^{1}$. Therefore, for any $n \geq 1, S_{n}$ is a bounded operator from $L^{p}(w)$ into itself, with norm not exceeding $n\|w\|_{1}^{1 / p}\left\|w^{-1 /(p-1)}\right\|_{1}^{1-1 / p}$. Since, by (1.3), $\sup _{n}\left\|S_{n} f\right\|_{p, w}<\infty$ for every $f \in L^{p}(w)$, (1.2) follows from the uniform boundedness principle. The proof of Theorem 1.1 is now complete.

\section{Proof of Theorem 1.2}

We shall prove the weak type $(1,1)$ result using Theorem 1.1 and the modified Calderón-Zygmund decomposition lemma obtained in [10].

Proof of (i) $\Rightarrow$ (ii). Suppose $w \in A_{1}(G)$. We have $L^{1}(w) \subset L^{1}$. It is sufficient to prove (1.5) with $S_{n}$ replaced by $S_{n}^{*}$. Let $f \in L^{1}(w)$. If $y<\|f\|_{1}$, then the $A_{1}(G)$ condition gives

$$
\int_{\left\{\left|S_{n}^{*} f\right|>y\right\}} w d \mu \leq\left(\int_{G} w d \mu\right) y^{-1} \int_{G}|f| d \mu \leq C y^{-1} \int_{G}|f| w d \mu .
$$

Therefore we can restrict ourselves to $y \geq\|f\|_{1}$.

Let $n=\sum_{k=0}^{\infty} \alpha_{k} m_{k}, 0 \leq \alpha_{k}<p_{k}$. From Lemma 2 of [10], we obtain a sequence of disjoint generalized intervals $\left\{I_{j}\right\}$ such that

$$
y<\frac{1}{\mu\left(I_{j}\right)} \int_{I_{j}}|f| d \mu \leq 3 y, \quad j=1,2, \ldots,
$$

and

$$
|f(x)| \leq y \quad \text { for a.e. } x \notin \bigcup_{j} I_{j} \equiv \Omega .
$$

Moreover, there is a decomposition of $f=g+b$, with $g, b \in L^{1}$, such that

$$
\begin{gathered}
g(x)=f(x) \quad \text { for } x \notin \Omega, \\
|g(x)| \leq \frac{C}{\mu\left(I_{j}\right)} \int_{I_{j}}|f| d \mu, \quad x \in I_{j}, \quad j=1,2, \ldots, \\
\int_{I_{j}} b d \mu=0, \quad j=1,2, \ldots,
\end{gathered}
$$

and

$$
\int_{I_{j}} b \phi_{k}^{\alpha_{k}} d \mu=0 \text { for all } I_{j} \in \mathscr{I}_{k}, k=0,1, \ldots
$$

(The constant $C$ in (5.4) is independent of $f$ and $y$.) 
Since

$$
\int_{\left\{\left|S_{n}^{*} f\right|>y\right\}} w d \mu \leq \int_{\left\{\left|S_{n}^{*} g\right|>y / 2\right\}} w d \mu+\int_{\left\{\left|S_{n}^{*} b\right|>y / 2\right\}} w d \mu,
$$

we shall show that each term on the right is bounded by $C y^{-1}\|f\|_{1, w}$.

Because $g \in L^{\infty} \subset L^{2}(w)$ and $w \in A_{1}(G) \subset A_{2}(G)$, it follows from Theorem 1.1 that

$$
\begin{aligned}
\int_{\left\{\left|S_{n}^{*} g\right|>y / 2\right\}} w d \mu & \leq 4 y^{-2} \int_{G}\left|S_{n}^{*} g\right|^{2} w d \mu \leq C y^{-2} \int_{G}|g|^{2} w d \mu \\
& =C y^{-2}\left(\int_{c_{\Omega}}|g|^{2} w d \mu+\sum_{j} \int_{I_{j}}|g|^{2} w d \mu\right) .
\end{aligned}
$$

By (5.3) and (5.2),

$$
\int_{c_{\Omega}}|g|^{2} w d \mu \leq y \int_{c_{\Omega}}|f| w d \mu
$$

Also, by (5.4), (5.1) and the $A_{1}(G)$ condition,

$$
\int_{I_{j}}|g|^{2} w d \mu \leq\left(\frac{C}{\mu\left(I_{j}\right)} \int_{I_{j}}|f| d \mu\right)^{2}\left(\int_{I_{j}} w d \mu\right) \leq C y \int_{I_{j}}|f| w d \mu .
$$

Substituting these estimates into (5.7), we obtain

$$
\int_{\left\{\left|S_{n}^{*} g\right|>y / 2\right\}} w d \mu \leq C y^{-1} \int_{G}|f| w d \mu .
$$

To estimate $S_{n}^{*} b$, let $\Omega^{*}=\bigcup_{j}\left(3 I_{j}\right)$. From $(2.1),(5.1)$ and the $A_{1}(G)$ condition, we have

$$
\begin{aligned}
\int_{\Omega^{*}} w d \mu & \leq C \sum_{j} \int_{I_{j}} w d \mu \\
& \leq C \sum_{j}\left(\int_{I_{j}} w d \mu\right)\left(y^{-1} \frac{1}{\mu\left(I_{j}\right)} \int_{I_{j}}|f| d \mu\right) \\
& \leq C y^{-1} \sum_{j} \int_{I_{j}}|f| w d \mu \leq C y^{-1} \int_{G}|f| w d \mu
\end{aligned}
$$

Hence, it is sufficient to show that

$$
\int_{\left\{x \notin \Omega^{*}:\left|S_{n}^{*} b(x)\right|>y / 2\right\}} w d \mu \leq C y^{-1} \int_{G}|f| w d \mu .
$$

To do this, we expand $S_{n}^{*} b$ as in (1.6) and (1.9). For $x \notin \Omega^{*}$, it follows from (5.3), (5.5) and (5.6) that the first three terms in (1.9) vanish. (See the explanation in [10, pp. 317-318].) Therefore, for $x \notin \Omega^{*}$, we have

$$
S_{n}^{*} b(x)=i \sum_{k=0}^{\infty}\left\{\phi_{k}^{-\alpha_{k}}(x) H_{k}\left(b \phi_{k}^{\alpha_{k}}\right)(x)-H_{k} b(x)\right\} .
$$

(5.8) will be proved if we can show

$$
\sum_{k=0}^{\infty} \int_{c_{\Omega^{*}}}\left|H_{k}\left(b \phi_{k}^{\alpha_{k}}\right)\right| w d \mu \leq C \int_{G}|f| w d \mu
$$


and

$$
\sum_{k=0}^{\infty} \int_{c_{\Omega^{*}}}\left|H_{k} b\right| w d \mu \leq C \int_{G}|f| w d \mu .
$$

Suppose $x \notin \Omega^{*}$. It follows from (1.8), (5.3), (5.5) and (5.6) that

$$
\begin{aligned}
H_{k}\left(b \phi_{k}^{\alpha_{k}}\right)(x)=\frac{1}{2} \frac{1}{\mu\left(G_{k}\right)} \sum_{\substack{I_{j} \subset x+G_{k} \\
I_{j} \in \mathscr{Y}_{k}}} \int_{I_{j}} b(t) \phi_{k}^{\alpha_{k}}(t) \\
\quad \times\left\{\cot \left(\frac{\pi\left(x_{k}-t_{k}\right)}{p_{k}}\right)-\cot \left(\frac{\pi\left(x_{k}-t_{k}^{j}\right)}{p_{k}}\right)\right\} d \mu(t),
\end{aligned}
$$

where $t^{j}=\left\{t_{k}^{j}\right\}_{k \geq 0}$ is any fixed point in $I_{j}$. (See [10, p. 318].) Let $I$ be any coset of $G_{k}$. Fubini's theorem gives

$$
\begin{aligned}
\int_{I \cap \Omega^{*}}\left|H_{k}\left(b \phi_{k}^{\alpha_{k}}\right)(x)\right| w(x) d \mu(x) \leq \frac{1}{2} \frac{1}{\mu(I)} \sum_{\substack{I_{j} \in I \\
I_{j} \in Y_{k}}} \int_{I_{j}}|b(t)| \\
\cdot \int_{I \cap c\left(3 I_{j}\right)}\left|\cot \left(\frac{\pi\left(x_{k}-t_{k}\right)}{p_{k}}\right)-\cot \left(\frac{\pi\left(x_{k}-t_{k}^{j}\right)}{p_{k}}\right)\right| w(x) d \mu(x) d \mu(t) .
\end{aligned}
$$

From the proof of Theorem 3.3 (see the estimate of $\left|E_{3}\right|$ below (3.9)), we have, for any $t \in I_{j}, I_{j} \subset I, I_{j} \in \mathscr{I}_{k}$,

$$
\frac{1}{\mu(I)} \int_{I \cap\left(3 I_{j}\right)}\left|\cot \left(\frac{\pi\left(x_{k}-t_{k}\right)}{p_{k}}\right)-\cot \left(\frac{\pi\left(x_{k}-t_{k}^{j}\right)}{p_{k}}\right)\right| w(x) d \mu(x) \leq C M w(t) .
$$

Moreover, the last term does not exceed $C w(t)$ for a.e. $t \in I_{j}$, by the $A_{1}(G)$ condition. Therefore

$$
\int_{I \cap \Omega^{*}}\left|H_{k}\left(b \phi_{k}^{\alpha_{k}}\right)\right| w d \mu \leq C \sum_{\substack{I_{j} \subset I \\ I_{j} \in \mathscr{Y}_{k}}} \int_{I_{j}}|b| w d \mu .
$$

Writing $b=f-g$, using (5.4) and the $A_{1}(G)$ condition again, we get

$$
\begin{aligned}
\int_{I_{j}}|b| w d \mu & \leq \int_{I_{j}}|f| w d \mu+\left(\frac{C}{\mu\left(I_{j}\right)} \int_{I_{j}}|f| d \mu\right)\left(\int_{I_{j}} w d \mu\right) \\
& \leq C \int_{I_{j}}|f| w d \mu .
\end{aligned}
$$

Substituting this into (5.11), and summing over all cosets $I$ of $G_{k}$, and then over all $k$, we obtain

$$
\sum_{k=0}^{\infty} \int_{c \Omega^{*}}\left|H_{k}\left(b \phi_{k}^{\alpha_{k}}\right)\right| w d \mu \leq C \sum_{k=0}^{\infty} \sum_{I_{j} \in \mathcal{Y}_{k}} \int_{I_{j}}|f| w d \mu \leq C \int_{G}|f| w d \mu .
$$

This proves (5.9).

(5.10) can be proved similarly, using (5.5) instead of (5.6). With this we have completed the proof of (i) $\Rightarrow$ (ii). 
Proof of (ii) $\Rightarrow$ (i). Suppose $w$ satisfies the conditions in (ii). We first prove (1.4) for $I=G$ and for $I=\mathscr{J}_{k}, k=0,1, \ldots$, with $\mu(I) \leq \mu\left(G_{k}\right) / 2$. Let $z>\operatorname{essinf}_{I} w$, and $E_{z}=\{x \in I: w(x)<z\}$. Then $\mu\left(E_{z}\right)>0$. Define $f=\chi_{E_{z}}$. We have $\|f\|_{1, w} \leq z \mu\left(E_{z}\right)<\infty$. Applying the proofs of (4.4) and (4.5) to this $f$, using (1.5) instead of (1.2), we get

$$
\int_{I} w d \mu \leq C \mu(I)\left(\int_{I} f d \mu\right)^{-1} \int_{I}|f| w d \mu \leq C \mu(I) z .
$$

Since this holds for any $z>\operatorname{essinf}_{I} w$, we have

$$
\frac{1}{\mu(I)} \int_{I} w d \mu \leq C \underset{I}{\operatorname{essinf}} w .
$$

This proves (1.4) for $I=G$, and for $I \in \mathscr{I}_{k}, k=0,1, \ldots$, with $\mu(I) \leq$ $\mu\left(G_{k}\right) / 2$. In particular, (1.4) holds for all cosets of $G_{k}, k=0,1, \ldots$.

For $I \in \mathscr{J}_{k}, k=0,1, \ldots$, with $\mu(I)>\mu\left(G_{k}\right) / 2$, let $I \subset x+G_{k}, x \in G$. Then

$$
\frac{1}{\mu(I)} \int_{I} w d \mu \leq \frac{2}{\mu\left(G_{k}\right)} \int_{x+G_{k}} w d \mu \leq 2 C \underset{x+G_{k}}{\operatorname{essinf}} w \leq 2 C \underset{I}{\operatorname{essinf}} w .
$$

This proves (ii) $\Rightarrow$ (i) and concludes the proof of Theorem 1.2.

\section{REFERENCES}

1. R. R. Coifman and C. Fefferman, Weighted norm inequalities for maximal functions and singular integrals, Studia Math. 51 (1974), 241-250.

2. A. Cordoba and C. Fefferman, $A$ weighted norm inequality for singular integrals, Studia Math. 57 (1976), 97-101.

3. C. Fefferman and E. M. Stein, $H^{p}$ spaces of several variables, Acta Math. 129 (1972), 137-193.

4. J. García-Cuerva and J. L. Rubio de Francia, Weighted norm inequalities and related topics, North-Holland, Amsterdam, 1985.

5. J. A. Gosselin, A weighted norm inequality for Vilenkin-Fourier series, Proc. Amer. Math. Soc. 49 (1975), 349-353.

6. R. Hunt, B. Muckenhoupt, and R. Wheeden, Weighted norm inequalities for the conjugate function and Hilbert transform, Trans. Amer. Math. Soc. 176 (1973), 227-251.

7. B. Muckenhoupt, Weighted norm inequalities for the Hardy maximal function, Trans. Amer. Math. Soc. 165 (1972), 207-226.

8. C. W. Onneweer, Multipliers on weighted $L_{p}$-spaces over certain totally disconnected groups, Trans. Amer. Math. Soc. 288 (1985), 347-357.

9. P. Simon, On a maximal function, Ann. Univ. Sci. Budapest. Eötvös Sect. Math. 21 (1978), 41-44.

10. W.-S. Young, Mean convergence of generalized Walsh-Fourier series, Trans. Amer. Math. Soc. 218 (1976), 311-320.

Department of Mathematics, University of Alberta, Edmonton, Alberta, Canada T6G 2G1 